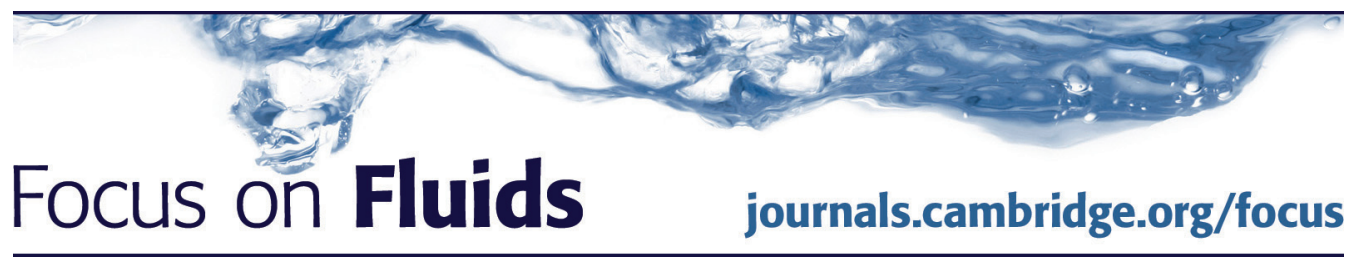

\title{
Turbulent patterns made simple?
}

\section{P. Manneville $\dagger$}

Hydrodynamics Laboratory, École Polytechnique, 91128 Palaiseau, France

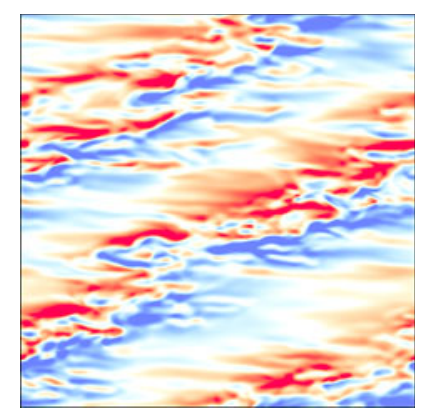

An observed, spectacular and puzzling distinctive feature of wall-bounded flows transitioning to/from turbulence is the large-scale ordered coexistence of laminar and turbulent flow over a wide range of Reynolds numbers. In their article, Chantry et al. (J. Fluid Mech., vol. 791, 2016, R8) circumvent the problem of turbulence modulation in genuine plane Couette flow by replacing no-slip boundary conditions at the walls with effective stress-free conditions constraining the interior part of the flow only. In so doing, they are able to collect relevant information on the laminar-turbulent patterning. The approach is then adapted to plane Poiseuille and pipe flow, giving to it a more universal value. Taking advantage of the stress-free conditions, they derive reduced models efficient at capturing the laminar-turbulent coexistence. Such models would be useful to interpret recent findings in the large-aspect-ratio limit.

Key words: low dimensional models, pattern formation, transition to turbulence

\section{Context}

Transitional wall-bounded flows are characterized by the presence of domains filled with turbulence separated from laminar flow by fluctuating interfaces. This coexistence, dubbed global subcriticality, is implied by the linear stability of the base flow at Reynolds numbers sufficiently high that non-trivial nonlinear solutions to the NavierStokes equations are equally possible. Remarkably, this property persists in different forms over a finite range of Reynolds numbers, $R \in\left[R_{g}, R_{t}\right]$, where $R_{g}$ is the threshold below which the base flow is unconditionally stable, and $R_{t}$ is another threshold above which a regime of uniform, featureless, turbulence prevails. Even more remarkably, in this intermediate $R$ regime, the laminar-turbulent alternation is spatially organized, a feature well described as 'barber pole turbulence' by Feynman reporting on earlier observations of a turbulent helix by Coles (1962) in cylindrical Couette flow.

How and when flows of this kind become turbulent and acquire their pattern has already been highlighted in the Focus on Fluids section. The earliest notice is that of Mullin (2010) pointing to experiments on plane Couette flow under

$†$ Email address for correspondence: manneville.jfm@ladhyx.polytechnique.fr 
rotation generalizing those of (Prigent 2001; Prigent et al. 2003), who first reported on multiple turbulent helices (bands) in cylindrical (plane) Couette flow. These experiments were performed at large aspect ratio, i.e. with streamwise and spanwise dimensions very large when compared to the gap. In contrast, most theoretical efforts have been exerted on flow configurations just large enough to allow for the development of chaotic flow, the so-called 'minimal flow unit' paradigm. On that basis, turbulence can be envisioned as a wandering of trajectories in the abstract phase space of the dynamical system to which the flow is assimilated, as emphasized by Cvitanović (2013). The approach however fails to account for the spatiotemporal behaviour observed in large-aspect-ratio systems since, by assumption, it cannot include large-scale modulations. The search for localized states in laterally unconstrained geometries highlighted by Eckhardt (2014) would be a step in the right direction, except that the laminar-turbulent alternation cannot be seen as a periodic arrangement of such weakly chaotic states while bands are much wider and filled with much more active turbulence.

The most obvious way to improve our empirical knowledge of pattern formation below $R_{t}$ is by performing numerical simulations of Navier-Stokes equations, but the large-aspect-ratio limit of interest to experiments readily makes them prohibitively expensive (Duguet, Schlatter \& Henningson 2010). Much effort has thus been dedicated to reduce their cost. The earliest successful attempt was by Barkley \& Tuckerman (2005), who considered plane Couette flow in a narrow but oblique domain aligned with the pattern's wavevector. Another way to reduce the computational load is to reduce the resolution in the non-extended directions. This possibility was first explored in pipe flow by Willis \& Kerswell (2009), who succeeded in capturing the essentials of its dynamics by restricting the number of azimuthal modes. A similar approach was later proposed by us and further systematically scrutinized in Manneville \& Rolland (2011) for plane Couette flow. There, a reduction of the wall-normal resolution proved efficient in reproducing the patterns and most of their qualitative properties.

The relative insensitivity of the patterning to small-scale dynamics then suggests the design of models in terms of Galerkin expansions of the primitive problem on a small enough set of well-chosen representative modes. Taking convection as an example, Lord Rayleigh (1916) explicitly introduced the recourse to stress-free boundary conditions to be able to solve the linearized problem without the cumbersome numerical computation of the no-slip case, which was done years later. His solution, in turn, served as a starting point for nonlinear Galerkin-type modelling of tremendous importance since it led to the Lorenz (1963) model focusing on chaotic temporal dynamics, on the one hand, and to the Swift \& Hohenberg (1977) model as a paradigm of pattern-forming systems. What worked for convection, the archetype of globally supercritical transition to turbulence, can also be tried to grasp the global subcriticality of transitioning wall-bounded flows, plane Couette flow to begin with. Rayleigh's analytical simplification was indeed chosen by Waleffe (1997) to introduce, via Galerkin expansion, his Lorenz-like model of eight nonlinear coupled ordinary differential equations governing the flow in minimal flow unit conditions, to which we proposed a Swift-Hohenberg like partial differential extension appropriate for patterns; see Manneville (2005) which also reviews the experimental background and the theoretical context.

The work of Chantry, Tuckerman \& Barkley (2016) under focus here (\$2) deals with the construction of realistic but economical representations of wall-bounded flows in transitional conditions, leading to reduced models of the kind sketched above, in view of really understanding pattern formation and decay in large-aspect-ratio subcritical systems $(\S 3)$. 


\section{Overview}

Relying on simulations of the flow configuration considered by Waleffe (1997), i.e. a shear flow between stress-free plates driven by a sinusoidal body force, Chantry et al. (2016) first observe a transition to turbulence via an oblique laminar-turbulent pattern strikingly similar to that of plane Couette flow. They further demonstrate that this qualitative analogy can be made more quantitative by placing effective stress-free boundary conditions at a strategic position inside the genuine plane Couette flow driven by the no-slip wall motion. This position corresponds to an empirically determined optimal matching of mean deviations from base flow in both systems, apart from boundary layers close to the plates where the physical no-slip condition applies. They support their proposal by detailed statistics on both flows within the Barkley-Tuckerman oblique domain framework. The programme is next completed by a standard Galerkin modelling stage truncated to contain seven fields, and numerical simulations showing that the so-obtained model also renders the bands. The authors then apply the same basic procedure to plane Poiseuille flow and Hagen-Poiseuille pipe flow: simulations in the featureless regime of the considered flows, localization of the strategic points in the turbulent mean flow profiles yielding the new relevant scales, and simulation of the corresponding appropriately forced stress-free interior flows. Additional symmetry conditions are however imposed in order to keep the base flow stable while competing with non-trivial solutions. Encouraging quantitative results are obtained from numerical simulations of these generalizations of Waleffe flow, for both the transitional-range width and the flow statistics.

\section{Perspectives}

Obtaining reliable information on the transition to turbulence in large-aspect-ratio wall-bounded flows is a delicate matter. The approach developed by Chantry et al. (2016) sketched above suggests that the flow in the no-slip boundary layers is enslaved to the dynamics in the interior flow. It confirms that, for Reynolds numbers well within the transitional range $\left[R_{g}, R_{t}\right]$, hydrodynamic fields are still sufficiently coherent to be described by a few amplitudes solely functions of the in-plane coordinates (on-axis in the pipe flow case). In turn, this strongly supports modelling attempts by truncation of wall-normal Galerkin expansions. Since models derived from the Navier-Stokes equations by such systematic expansions, including those of Chantry et al., already all have the same structure (Seshasayanan \& Manneville 2015), introducing the notion of 'interior flow' explicitly gives the theory more physical content. On the one hand, this should be a invitation to play with the coefficients in the generic model to get insight into what controls the pattern's main characteristics, i.e. angle, wavelength and width of the laminar-turbulent coexistence range. On the other hand, we should also try to understand the role of what is left out by the interior-flow assumption - the dynamics inside the boundary layers - and can be restored on a case-by-case basis by an increase of the order of truncation.

In any case, optimally reduced models could also be used to further explore the whole transitional-range span. The computational parsimony of such models would permit the study of the 'thermodynamic limit' (wide systems, long times) in view of addressing some open questions: (i) Accounting for wavelength selection of the emerging pattern below $R_{t}$ and its orientational fluctuations. Direct numerical simulations in the quasi-two-dimensional case (plane Couette or Poiseuille flow) can constrain the pattern away from a possibly optimal configuration if the domain is too small and it may be difficult to achieve statistical equilibrium in the long-time limit. 
(ii) Determining whether the turbulent fraction for decay at $R_{g}$ varies continuously or discontinuously. At present, the best evidence that this behaviour is continuous with exponents, i.e. 'critical' in the sense of statistical physics, in a quasi-one-dimensional Couette geometry (Lemoult et al. 2016) whereas under-resolved numerics in the quasi-two-dimensional case speak in favour of a discontinuous transition (Manneville 2011).

Finally, on the analytical side, simplifying the models from their generic expressions could help us better understand wall-bounded transitional mechanisms. Demonstrating the general relevance of the reaction-diffusion scheme (Barkley 2011; Manneville 2012) by systematic approaches based on the Galerkin approximation would be of much interest in this respect.

\section{References}

Barkley, D. 2011 Simplifying the complexity of pipe flow. Phys. Rev. E 84, 016309.

BArKley, D. \& TuCKerman, L. S. 2005 Computational study of turbulent laminar patterns in Couette flow. Phys. Rev. Lett. 94, 014502.

Chantry, M., Tuckerman, L. S. \& Barkley, D. 2016 Turbulent patterns in shear flows without walls. J. Fluid Mech. 791, R8.

COLES, D. 1962 Interfaces and intermittency in turbulent shear flow. In Mécanique de la Turbulence (ed. A. Favre), pp. 229-248. CNRS.

CVitanović, P. 2013 Recurrent flows: the clockwork behind turbulence. J. Fluid Mech. 726, 1-4.

ECKhardT, B. 2014 Doubly localized states in plane Couette flow. J. Fluid Mech. 758, 1-4.

Duguet, Y., Schlatter, P. \& Henningson, D. S. 2010 Formation of turbulent patterns near the onset of transition in plane Couette flow. J. Fluid Mech. 650, 119-129.

Lemoult, G., Shi, L., Avila, K., Jalikop, S. V., Avila, M. \& Hof, B. 2016 Directed percolation phase transition to sustained turbulence in Couette flow. Nature Phys. 12, 254-258.

LORD RAYLEIGH 1916 On convection currents in a horizontal layer of fluid, when the higher temperature is on the under side. In Scientific Papers, vol. 6, 1911-1019, pp. 432-446. Cambridge University Press, 1920.

Lorenz, E. N. 1963 Deterministic nonperiodic flow. J. Atmos. Sci. 20, 130-141.

Manneville, P. 2005 Modeling the direct transition to turbulence. In IUTAM Symposium on Laminar-Turbulent Transition and Finite Amplitude Solutions (ed. T. Mullin \& R. R. Kerswell), pp. 1-33. Springer.

Manneville, P. \& Rolland, J. 2011 On modelling transitional turbulent flows using under-resolved direct numerical simulations: the case of plane Couette flow. Theor. Comput. Fluid Dyn. 25, $407-420$.

Manneville, P. 2011 On the decay of turbulence in plane Couette flow. Fluid Dyn. Res. 43, 065501.

Manneville, P. 2012 Turbulent patterns in wall-bounded flows: a Turing instability? Eur. Phys. Lett. 98, 64001.

Mullin, T. 2010 The rich structure of transition in a shear flow. J. Fluid Mech. 648, 1-4.

Prigent, A. 2001 La spirale turbulente: motif de grande longueur d'onde dans les écoulements cisaillés turbulents. PhD thesis, University Paris-XI. See tel.archives-ouvertes.fr/tel-00261190.

Prigent, A., Grégoire, G., Chaté, H. \& Dauchot, O. 2003 Long-wavelength modulation of turbulent shear flows. Physica D 174, 100-113.

Seshasayanan, K. \& Manneville, P. 2015 Laminar-turbulent patterning in wall-bounded shear flow: a Galerkin model. Fluid Dyn. Res. 47, 035512.

Swift, J. \& Hohenberg, P. C. 1977 Hydrodynamic fluctuations at the convective instability. Phys. Rev. A 15, 319-328.

Waleffe, F. 1997 On a self-sustaining process in shear flows. Phys. Fluids 9, 883-900.

Willis, A. P. \& Kerswell, R. R. 2009 Turbulent dynamics of pipe flow captured in a reduced model: puff relaminarization and localized 'edge' states. J. Fluid Mech. 619, 213-233. 